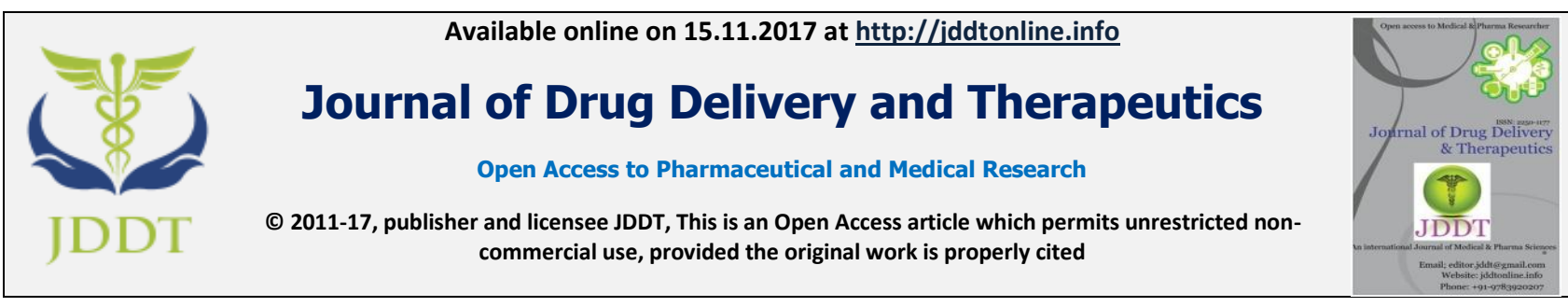

Open $\bigcirc$ Access

Review Article

\title{
A ROLE OF MACROPHAGES: AN OVERVIEW
}

\author{
Nikita Verma, Swarnlata Saraf \\ University Institute of Pharmacy, Pt. Ravishankar Shukla University Raipur, (C.G.) 492010, India
}

\begin{abstract}
The macrophage is a type of phagocytic cell, which is a type of cell those are responsible for detecting, engulfing and destroying pathogens and apoptotic cells. Macrophages are produced through the differentiation of monocytes, which turn into macrophages when they leave the blood. Macrophages also play a role in alerting the immune system to the presence of invaders. Macrophage lineage cells present a remarkably versatile array of functional specializations across vertebrates. As resident cells in virtually all tissues, macrophages aid in maintaining homeostatic environments, and upon infection, are typically one of the first cell types to encounter intruding pathogens, where they orchestrate appropriate immune responses. Another function of macrophages is to alert the immune system to microbial invasion. After ingesting a microbe, a macrophage presents a protein on its cell surface called an antigen, which signals the presence of the antigen to a corresponding T helper cell. Moreover macrophages might be a successful targeting site for targeted drug delivery approaches.
\end{abstract}

Keywords: macrophages, phagocytic cells, monocytes, immune system

Article Info: Received 03 Sep, 2017; Review Completed 17 Oct, 2017; Accepted 05 Nov, 2017; Available online 15 Nov, 2017

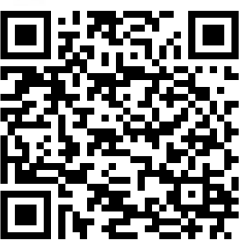

Cite this article as:

Verma N, Saraf S, A role of macrophages: an overview, Journal of Drug Delivery and Therapeutics. 2017; $7(6): 91-103$

DOI: http://dx.doi.org/10.22270/jddt.v7i6.1521

*Address for Correspondence

Swarnlata saraf, University Institute of Pharmacy, Pt. Ravishankar Shukla University Raipur, (C.G.) 492010, India. Email: Swarnlata_saraf@rediffmail.com

\section{INTRODUCTION}

A type of cell derived fromthat ingests (takes in) foreign material.Is a kind of swallowing cell, which means it functions by literally swallowing up other particles or smaller cells Taken from Greek Words (Greek: big eaters, from makros "large" + phagein "eat"; abbr. MФ). Human macrophages are about 21 micrometres (0.00083 in) in diameter. Monocytes and macrophages are phagocytes, acting in both non-specific defense (and innate immunity) as well as to help initiate specific defence mechanisms (or adaptive immunity) of vertebrate animals. They move by action of Amoeboid movement.Life time depends on the type of tissue, viability ranges between 6 and 16 days. Macrophage lineage cells present a remarkably versatile array of functional specializations across vertebrates. As resident cells in virtually all tissues, macrophages aid in maintaining homeostatic environments, and upon infection, are typically one of the first cell types to encounter intruding pathogens, where they orchestrate appropriate immune responses ${ }^{1}$. Macrophages were first identified by Elie Metchnikoff as phagocytic cells which helped to eliminate pathogens in both invertebrates and vertebrates ${ }^{2}$. In 1905, his research findings suggested that macrophages from infected animals had elevated ability of killing bacteria, thereby proposing the basis of the concept of macrophage activation ${ }^{1}$.Our understanding of macrophage biology has greatly expanded since the first description of starfish larvae phagocytes in 1882 by Élie Metchnikoff, who was later awarded the Nobel Prize for his contribution to cellular immunity in $1908^{3}$.

Much of our understanding of macrophage biology comes from research in mammalian models, where distinct macrophage subsets of have been characterized, including classically activated cells by interferons (IFN) and tumor necrosis factor alpha (TNF $\alpha$ ) (M1); alternatively activated cells by IL-4 and IL-13 (M2a); 
macrophages activated by immune complexes or apoptotic cells (M2b); and regulatory macrophages, deactivated by IL-10, TGF- $\beta$, or glucocorticoids (M2c), which culminate in the various effector subtypes, broadly described as having a "kill" or "heal" response (reviewed in) ${ }^{4}$. Recent transcriptomic and proteomic analyses of macrophage populations derived by these distinct stimuli have underlined the vast complexities of these mechanisms at the molecular level, controlling the various physiological responses of macrophages ${ }^{5}$.

In teleosts, the best characterized macrophage phenotype is that comparable to the M1 activation state, which serves a critical role in host protection. These cells may rapidly kill pathogens by engulfment and production of toxic reactive intermediates, phagolysosomal acidification, and restriction of nutrient availability ${ }^{6,7}$. Furthermore, M1 macrophages are robust factories of cytokines, chemokines, and lipid mediators, which act to potentiate and fine-tune the inflammatory and adaptive immune responses. More recently, efforts to characterize alternative activation states of teleost macrophages are focusing on the biology of fish IL4 and IL13 homologues (IL4/13A and IL4/13B) and arginase activity, implying a conserved M2a phenotype ${ }^{7}$. Similar deactivating roles of glucocorticoids (GC), immune complexes, IL-10, and TGF- $\beta$ have been demonstrated in teleosts, suggesting conserved functions of these ligands in deactivating or aiding in the tissue repair ${ }^{8,9}$. Characterization of the regulatory mechanisms responsible for shaping macrophage polarity is a unique challenge in teleosts, as whole-genome duplication and gene-specific duplication events, combined with disparate evolutionary pressures, have endowed distinct teleosts with multiple gene copies, the product of some of which may have adopted respectively divergent roles 10,1

Although several attempts have been made to classify macrophages, the most successful definition is the mononuclear phagocytic system (MPS), which encompasses these highly phagocytic cells and their bone marrow (BM) progenitors. In the MPS schema, adult macrophages are defined as end cells of the mononuclear phagocytic lineage with tissue macrophages deriving from circulating monocytes that originate in the BM. However, this definition is inadequate as macrophages have several origins during ontogeny and each of these different lineages persist into adulthood where they display great diversity ${ }^{12,13}$.

Other classifications have included binary classifications that refer to inflammatory status. These include the activated Macrophages (AM) and alternatively activated Macrophages (AAM) or M1 or M2 defined by responses to the cytokines IFN $\gamma$ and activation of TLRs and IL4/IL13 respectively. While this is a useful heuristic that may reflect extreme states such as in AMs in THI mediated immune responses or AAM in parasitic infections .it cannot represent the complex in vivo milieu for most macrophage types where numerous cytokines and growth factors interact to define the final differentiated state of macrophages. Indeed transcriptional profiling of resident macrophages by the "Immunological Genome Project" finds that these populations show great transcriptional diversity with minimal overlap suggesting many unique classes. Macrophages have roles in almost every aspect of an organism's biology ranging from development, homeostasis, to repair through to immune responses to pathogens ${ }^{14,15}$. Resident macrophages regulate tissue homeostasis by acting as sentinels and responding to changes in physiology as well as challenges from outside. During these homeostatic adaptations, macrophages of different phenotypes can also be recruited from the monocyte reservoirs of blood, spleen and bone marrow, and perhaps, from resident tissue progenitors or through local proliferation. Unfortunately, in many cases, these homeostatic and reparative functions can be subverted by continuous insult, resulting in causal association of macrophages with disease states, such as fibrosis, obesity and cancer (Figure 1). Macrophages, therefore, are an incredibly diverse set of cells constantly shifting their functional state to new set points in response to changes in tissue physiology or environmental challenges. They should not even be considered as one cell type but be subdivided into different functional subsets with acknowledgement to their different origin ${ }^{16}$.

In addition to IL-4/IL-13, a great number of stimuli, such as antibody immune complexes together with lipopolysaccharide (LPS) or IL-1, transforming growth factor- $\beta$ (TGF- $\beta$ ), glucocorticoids and IL-10, were found to have the ability of alternative activation of macrophages ${ }^{17}$. As they shared properties with IL-4/IL13-activated macrophages, a new functional state called M2-like phenotype was proposed, and it held great promise for the research of macrophage activation in a dynamic microenvironment (Figure 1). M1 phenotype macrophages express numerous pro-inflammatory mediators including tumor necrosis factor (TNF)- $\alpha$, IL1, IL-6, reactive nitrogen and oxygen intermediates, which have a strong microbicidal and tumoricidal activity; while M2 phenotype express molecules including resistin-like- $\alpha$ (also known as Fizz1), Arginase1 (Arg1), chitinase 3-like 3 (also known as Ym1), IL-10 and Mrc1 (also known as CD206), which are supposed to be involved in parasite infestation, tissue remodeling and tumor progression (immunoregulatory functions $)^{18}$. M1 and M2 phenotype macrophages can be converted into each other in their specific microenvironment, and they are quite different with Th1 and Th2. Many key transcription factors are involved in macrophage polarization, like signal transducer and activator of transcription (STATs), interferon-regulatory factor (IRFs), nuclear factor (NF)- $\kappa \mathrm{B}$, activator protein (AP) , peroxisome proliferator- activated receptor (PPAR)- $\gamma$ and cAMP-responsive element-binding protein (CREB), which interact with each other and regulate macrophages to certain phenotype in the various inflammatory diseases (Figure 2). Here we briefly review the polarization of macrophages and their functions in some typical inflammatory diseases ${ }^{19}$. 
- Phagocytic Mononuclear cells were more adept at killing bacteria after resistance to infection, which set the concept of "macrophage activation"

- This mechanism in killing bacteria of macrophages were gradually reveled

- Lymphocytes were identified as the major antigen-specific cells responsible for macrophage microbicidal activation.

- IFN- $\gamma$ was discovered as the key factor of antimicrobial macrophage activation, known as classical activation

- Discovery of Th1 and Th2

- The respiratory burst was inhibited and the production of IL-1 $\beta$ was decreased in the $\mathrm{IL}-4$ and IL-13 mediated immune responses

- The alternative activation of macrophage was proposed with the finding of upregulation of MRC1 as a distinctive marker

- The 'spectrum' of macrophage activation states was proposed

- The concept of M2- like state was raised, which contributed to a new classification of microphage activation .

Figure 1: Timeline: advances in research of macrophage polarization

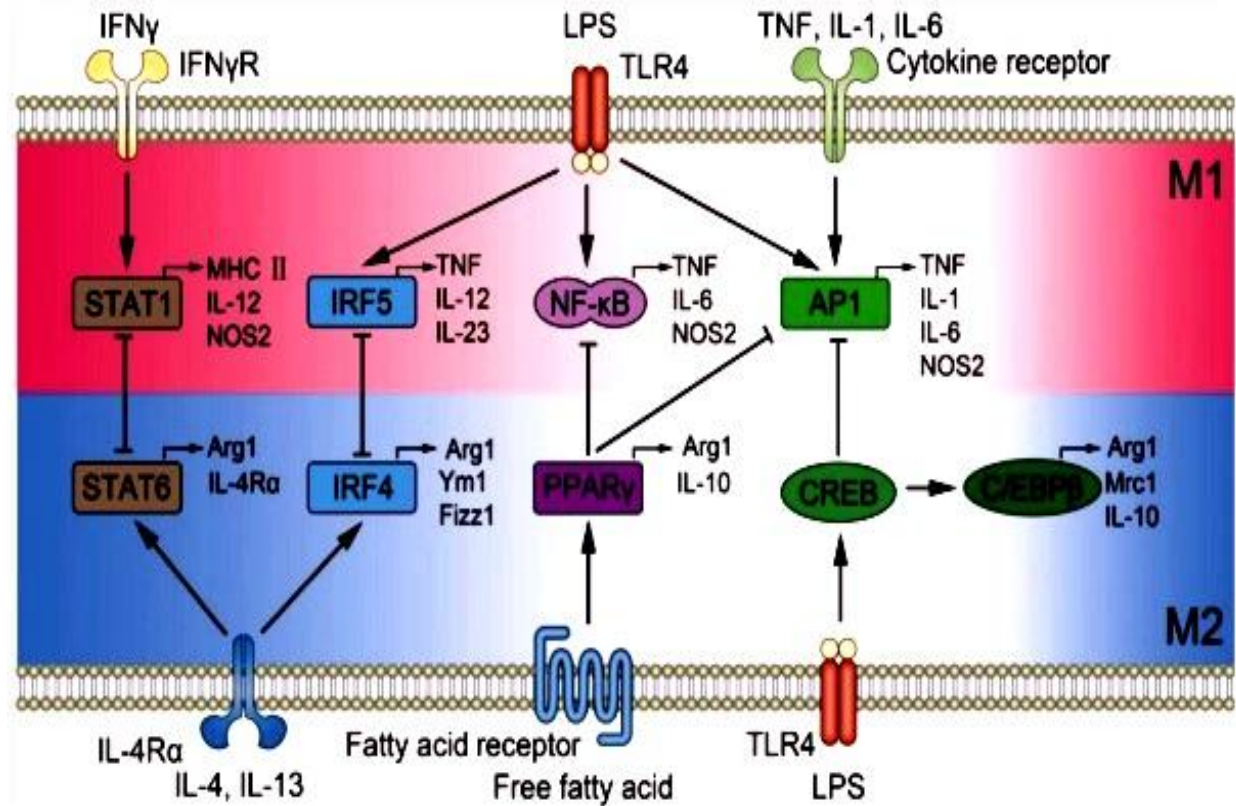

Figure 2: Signal pathways of macrophage polarization. The figure illustrates several mechanisms underlying macrophage polarization and shows the feedback regulation between M1 and M2 signal pathways. Those include the activation of STAT1 mediated by IFN- $\gamma$ receptor, increase in IRF5, NF- $\kappa$ B, as well as AP1 expression mediated by Toll-like receptor 4 (TLR4), enhanced AP1 expression mediated by cytokine receptor, activation of STAT6 and increased IRF4 mediated by IL-4 receptor, increased level of PPAR $\gamma$ mediated by fatty acid receptor, and enhanced expression in CREB by TLR4. The feedback regulation between M1 and M2 are implemented by STAT1-STAT6, IRF5-IRF4, NF- $\mathrm{kB}-\mathrm{PPAR} \gamma$, AP1CREB, and AP1-PPAR $\gamma$, and they play essential roles in the initiation, development, and cessation of inflammatory diseases.

\section{From monocyte to macrophage}

The chemicals secreted by damaged cells, mast cells; high conc. of LDL; bacterial by products etc. promote upregulation of adhesion molecules on the endothelial cells (EC). Thus monocyte and lymphocyte recruitmentis initiated. Upregulated exposure of adhesion molecules, and chemoattractant production and release are essential elements of the transfer of monocytes to the intima and the concurrent differentiation of these cells into macrophages. Thus the differentiation of monocytes into macrophages is a chemical process. $^{20}$

\section{Phagocytosis (Paracrine)}

Removal of necrotic cell debris from lungs, spleen, bone, liver, neural tissue is one very important task of macrophages. This is done by fixed macrophages from connective tissue -histiocytes \& giant cells.

$\checkmark \quad$ liver sinusoids - Kupffer's cells

$\checkmark \quad$ lung - alveolar macrophages / Dust cells

$\checkmark \quad$ lymph nodes - free and fixed macrophages 
$\checkmark \quad$ spleen - sinusoidal lining cells

$\checkmark$ bone marrow - osteoclast/osteoblast

$\checkmark \quad$ serous fluids - pleural and peritoneal macrophages

$\checkmark \quad$ skin - histiocytes, Langerhans's cell

\section{Adaptive Immunity}

When a macrophage ingests a pathogen, the pathogen becomes trapped in a phagosome, which then fuses with a lysosome. Macrophages can digest more than 100 bacteria before they finally die due to their own digestive compounds. After digesting the pathogen, macrophage will present the antigen of the pathogen to the corresponding helper $\mathrm{T}$ cell. The presentation is done by integrating it into the cell membrane and displaying it attached to an MHC class II molecule, indicating to other white blood cells that the macrophage is not a pathogen, despite having antigens on its surface.

\section{Muscle Regulation ${ }^{20,21}$}

This happens in multiple steps. The first wave of phagocytic macrophages degrades and engulfs the injured muscle fibres and the debris. (During times of increased muscle usage) (ED1+)The second wave of non-phagocytic macrophages spreads among the muscle fibres and releases chemicals which stimulate proliferation of muscles. (ED2+) (It is thought that macrophages release soluble substances that influence the proliferation, differentiation, growth, repair, and regeneration of muscle, but at this time the factor that is produced to mediate these effects is unknown. It is known that macrophages' involvement in promoting tissue repair is not muscle specific; they accumulate in numerous tissues during the healing process phase following injury.) Suspected factors: Thrombospondin1, TGF-alpha.

\section{TYPES OF MACROPHAGES (ON THE BASIS OF ACTIVATION MECHANISM)}

$\checkmark \quad$ M1 (classically activated macrophages)

$\checkmark$ Pro-inflammatory phenotype (accelerate inflammation)

$\checkmark$ Activated by type 2 interferon (IFN-gamma) and tumor necrosis factor(alpha)

$\checkmark \quad$ Released by all due to presence of pathogens

$\checkmark \quad$ M2 (alternatively activated macrophages)

Anti-Inflammatory Phenotype

$\checkmark$ Activated by Interleukin- 4, interferon type -1(IFNalpha)

$\checkmark \quad$ Released by CD4+ T cells (helper T cell)

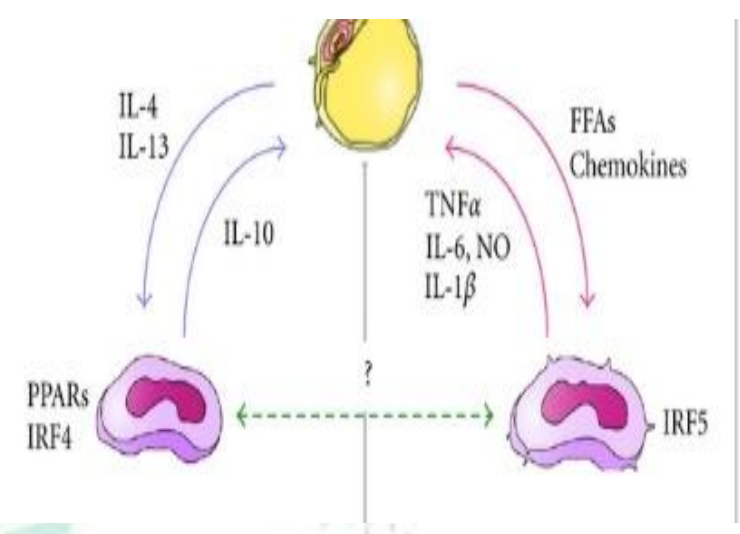

Figure 3: Anti-Inflammatory Phenotype

Types of macrophages

Differentiation of monocyte

(irf $=$ interferon regulatory facots, ppar=Peroxisome proliferator-activated receptor)

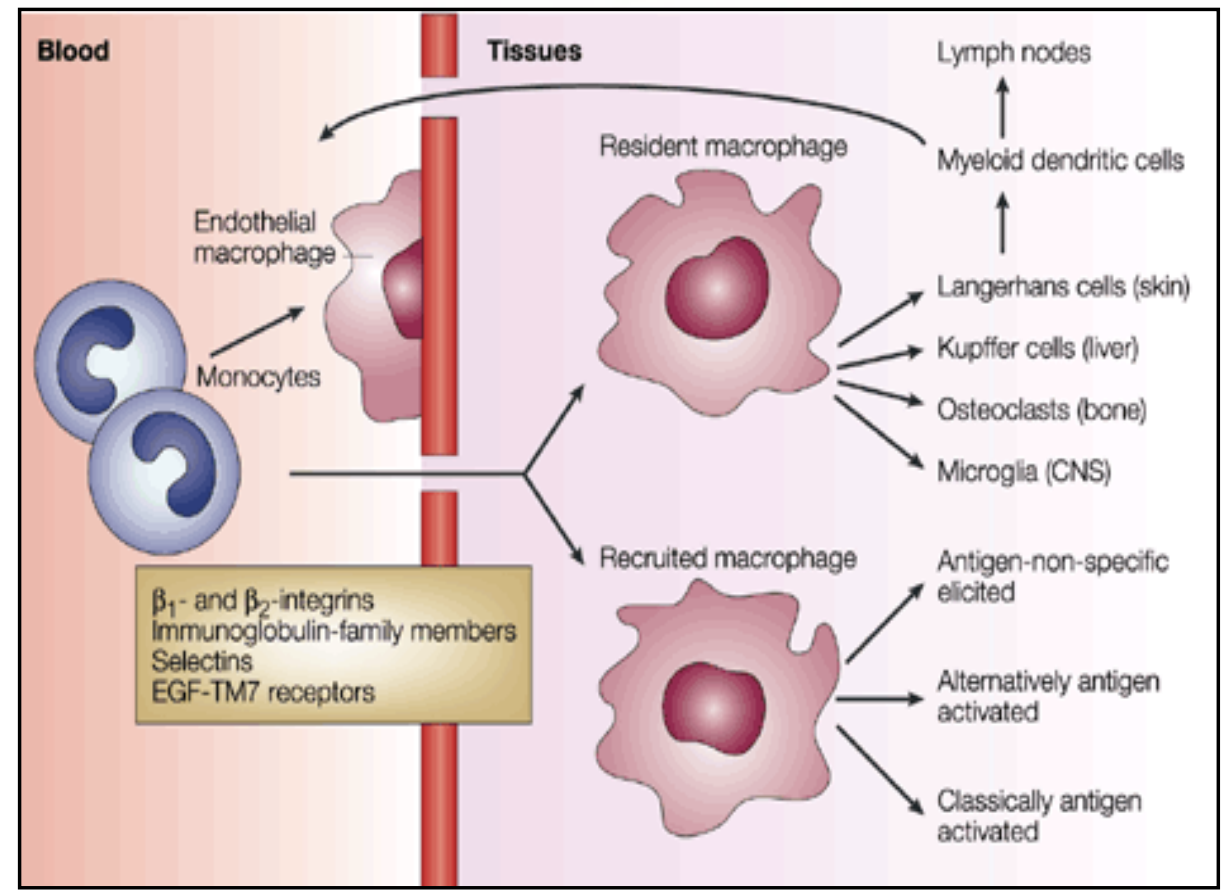

Figure 4: Macrophage Localisation 
Macrophages in Inflammation

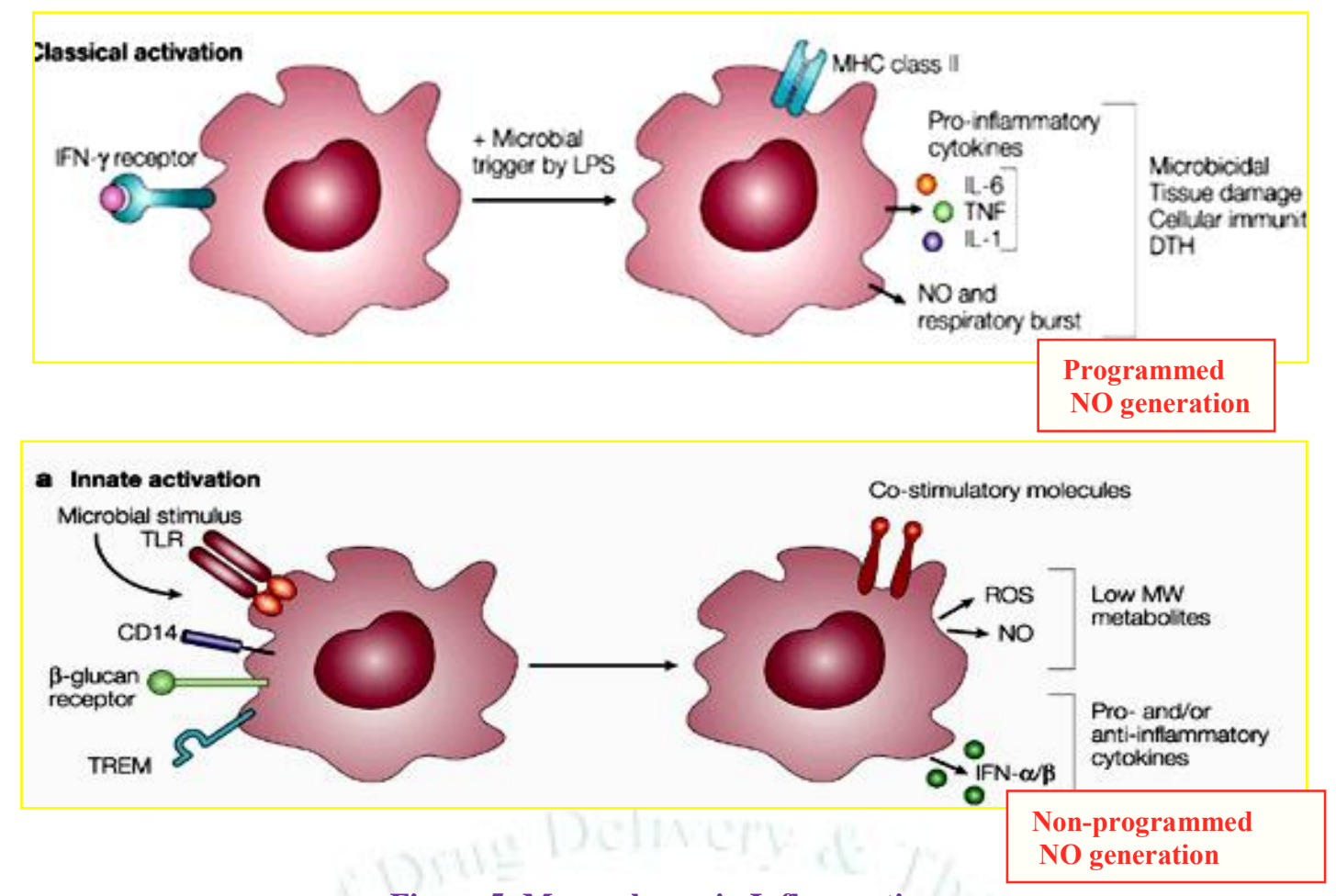

Figure 5: Macrophages in Inflammation

\section{Formation of macrophage}

The blood monocytes are young cells (immature macrophage) that already possess migratory, chemotactic, pinocytic and phagocytic activities. Under migration into tissues, monocytes undergo further differentiation (at least one day) to become multifunctional tissue macrophages.

Macrophage Exhibit 3 main functions in body:

1. Destroy bacteria by phagocytosis

2. Activate other immune function

3. Phagocytose apoptotic cells

\section{Phagocytosis}

Once it leaves blood vessel and migrated to tissue, the next job is to EAT the pathogen. This human macrophage, like neutrophil, is a professional "phagocyte" or eating cell (phago = "eating", cyte = "cell"). Furthermore, the pathogen will be digested by using enzyme from macrophage, in the end resulting antigen and waste material.

Steps of phagocytosis

1. A phagosome is formed to ingest the pathogen

2. Lysosome + phagosome $\rightarrow$ phagolysosome
3. Pathogen is digested by using enzymeand toxic (such as superoxideanion or nitric oxide) Secreting waste material.

Perform specific immune function ${ }^{26}$

- After digesting a pathogen, present antigen (identification)

- Churn out powerful chemical substances (monokines) including enzymes and complement proteins.

- The presentation is done by integrating it into the cell membrane, indicating to other

- White blood cells that the macrophage is not a pathogen, despite having antigens on its surface.

- Antigen presentation results in the production of antibodies that attach to the antigens of pathogens, making them easier for macrophages to adhere to with their cell membrane and phagocytose.

- Secrete hormones cytokines

- To attract system immune cells to the site and activate cells involved in tissue repair to send signaling path to injury site.

Phagocytose apoptotic cells

These reduce the potential for an inflammatory response by ensuring that the dying cells are cleared before their intracellular contents have released. 


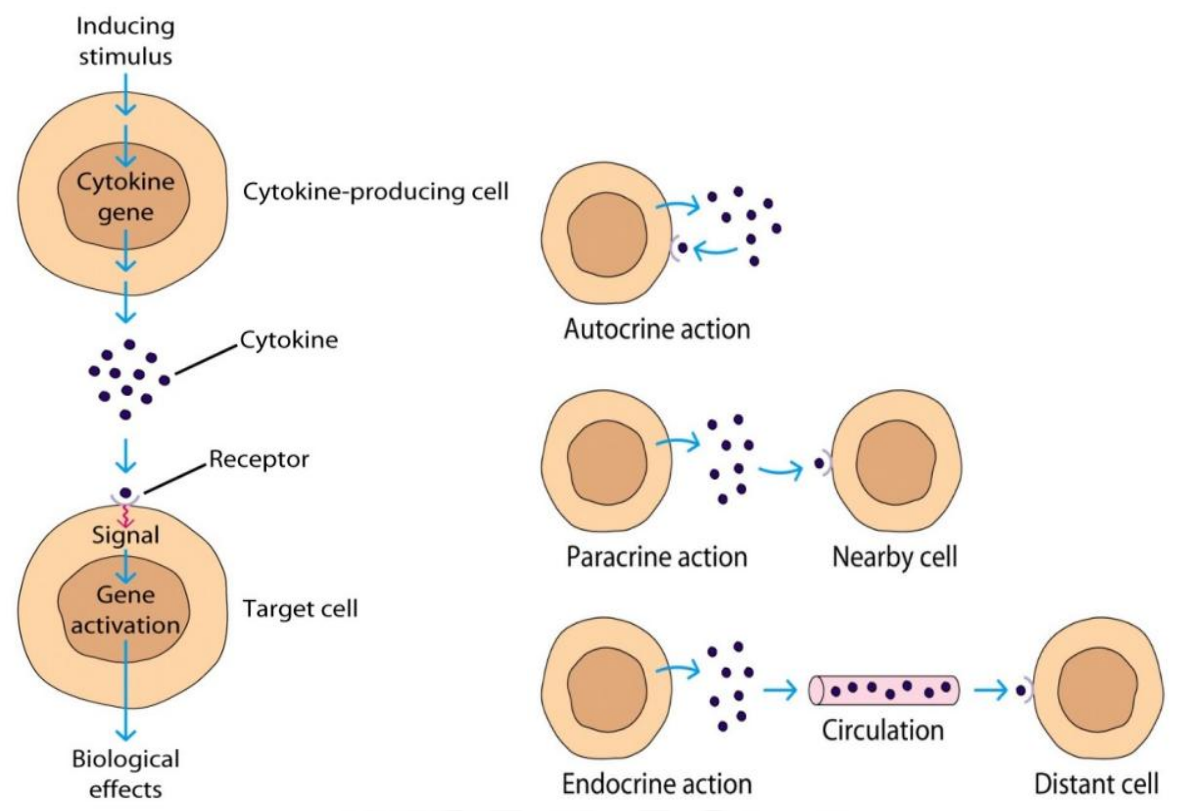

Figure 6: Function of Macrophage

\section{Inflammatory macrophages}

present in various exudates (developmental stage and not the functional state) Derived exclusively from monocytes, thus similar properties .

\section{Mechanisms:}

$\checkmark \quad$ Intake of monocytes from the circulating blood,

$\checkmark \quad$ Local proliferation and

$\checkmark$ Biological turnover

$\checkmark$ Under Normal Steady-State Conditions, The Renewal Of Tissue Macrophages Occurs Through Local Proliferation Of Progenitor Cells And Not Via Monocyte Influx.

\section{Mechanism of inflammation}

It is"dynamic response of vascularized tissues to injury" It is a complex multi-step process of tissue response to injury

\section{Purposes:}

To defend against injurious agentStart healing \& repair of injured tissue (bring defence forces such as WBC, antibodies, more nutrients and healing factors to the site of injury) Localizes infection and prevents spread.

\section{Chemical mediators :}

Chemical substances synthesised or released which mediate the changes in inflammation.

- Histamine by mast cells - vasodilatation.

- Prostaglandins - Cause pain \& fever.

- Bradykinin - Causes pain.

Clinical signs of inflammation (due to vascular permeability which allows for phagocytic chemotaxis)

$>$ Lewis triple response

$\checkmark \quad$ Flush (redline) : capillary dilatation $\checkmark \quad$ Flare (red zone) : arteriolar dilatation

$\checkmark$ Weal (edema) : exudation

$>$ Classic five signs

$\checkmark$ Rubor(redness)

$\checkmark$ Tumor(swelling)

$\checkmark$ Calor(Heat)

$\checkmark$ Dolor (pain)

\section{$\checkmark \quad$ Loss of function}

Macrophages have numerous different functions that may be injurious or reparative. Key issues for the future to devise a more complete understanding of the range of macrophage activities, how to identify macrophage "phenotypes" in vivo and how to manipulate macrophage function as therapy.

\section{Biological functions of macrophages}

Macrophages are involved at all stages of the immune response. First, as already outlined, they act as rapid protective mechanism which can respond before $\mathrm{T}$ cellmediated amplification has taken place. Activated macrophages play a key role in host defence against intracellular parasitic bacteria, pathogenic protozoa, fungi and helminths as well as against tumours, especially metastasing tumours. After phagocytosis, macrophages prevent intracellularly parasitic organisms from replication at least by three ways; Intracellular environment is unsuitable for microbial reproduction due to low $\mathrm{pH}$ and lack of nutrients in a phagolysosome .The toxic reaction may be activated to against dividing organisms. This include ROI, hypochlorite, NO myeloperoxidase, neutral proteases and lysosomal hydrolases Macrophages may also produce microbiostatic effector molecules at a steady-state and thus maintain intracellular microorganisms in the nonreplicating state. This latent infection is generally observed only in such individuals whose macrophages 
cannot be sufficiently activated. Generally, macrophages represent the second line of defence against different agent.

In addition, macrophages are important killer cells ( $\mathrm{K}$ cells); by means of antibody-dependent cell-mediated cytotoxicity (ADCC) they are able to kill or damage extracellular targets. They also take part in the initiation of $\mathrm{T}$ cell activation by processing and presenting antigen. Finally they are central effector and regulatory cells of the inflammatory response. To fulfil these functions, macrophages in their activated state are able to produce more than one hundred of different substances.

\section{Role of Macrophages in inflammation}

Inflammation is a process carried out in response to either physical or immunological tissue insults. It consists of a destructive process to remove the inflammatory trigger and damaged tissue, followed by repair and replacement ${ }^{20}$. The main actors in the destructive phase are neutrophils and macrophages Inflammation is signaled by heat, pain, redness, and swelling. Inflammatory conditions are named with the suffix itis .Macrophages are highly complex cells engaged in normal tissue maintenance and turnover among their varied functions, they are sentinels against infection, both through the use of receptors for common molecules carried by pathogens, and through opsonization. Macrophages exist in various levels of activation, wherein they become more aggressive at destroying macromolecules, and sending distress signals to recruit other components of the inflammatory response. The most aggressively activated macrophages are derived from blood monocytes in response to inflammatory signals. These are called "infiltrating macrophages $^{21}$.

Names of macrophages according to tissue locations ${ }^{22}$

\begin{tabular}{|l|l|l|}
\hline S. N. & Name & Location \\
\hline 1. & Connective tissue & Histiocyte \\
\hline 2. & Serous cavity & Peritoneal Macrophages \\
\hline 3. & Liver & Kupffer cell \\
\hline 4. & Bone Tissue & Osteoclast \\
\hline 5. & Lungs & $\begin{array}{l}\text { Alveolar Macrophages } \\
\text { (Dust Cell) }\end{array}$ \\
\hline 6. & Nervous System & Microglial cell \\
\hline 7. & Spleen & Sinusoidal Lining Cell \\
\hline 8. & Skin & Langerhans cell \\
\hline 9. & Inflamed Tissue & Infiltrating Macrophahge \\
\hline
\end{tabular}

\section{Tissue macrophages}

Macrophages can be thought of as a dispersed homeostatic organ Tissue macrophages constitute a distributed mononuclear phagocyte cellular system (MPS), contributing to the body's responses to physiologic changes and to infectious challenge; thus, the MPS is comparable to the nervous and endocrine systems, in that it is adaptable, regulated and able to perform trophic as well as defence functions, locally and systemically $^{26}$. Local macrophages induce tissuespecific metabolic responses such as hepatocyte biosynthesis of plasma proteins that provide an early response to infection in the acute phase reaction, and initiate features of systemic inflammation and infection such as loss of appetite and tissue catabolism ${ }^{27}$. The dual nature of macrophage functions, host protection versus tissue injury, is maintained in a fine balance; broadly, macrophage phagocytosis, clearance and secretion contribute to innate and adaptive defences against infection and underpin the process of inflammation, while the same processes, but with distinct secreted signals, restore tissue homeostasis and promote subsequent repair ${ }^{28}$.

Myeloid cells of the MPS interact with cells of the lymphoid system at many levels, recognition of non-self or modified self-antigens, initiating cellular and antibody immune responses, while executing effector functions which, if excessive or perpetuated, bring about tissue destruction. Monocyte migration and widespread tissue distribution provide portals for microbial dissemination, as well as host protection. During malignancy, tissue macrophages play an important role in promoting the survival, growth and spread of tumour cells ${ }^{29}$.

\section{Tumor-associated macrophages (TAM)}

Tumors engage the immune system from their inception. Initially, this mainly involves cells of the innate system such as macrophages and mast cells with their prevalence dependent on tumor type. However, even early on, there is also engagement of cells of the acquired system-particularly $\mathrm{T}$ cells (Gajewski et al., 2013). Nevertheless, despite this adaptive response and data that suggest better prognosis with $\mathrm{CD} 8+\mathrm{T}$ cell infiltration in some cancers, there is little evidence of immune rejection in established tumors, arguing that the local tumor microenvironment is immunosuppressive (Gajewski et al., 2013). Macrophages are among the most abundant normal cells in the tumor microenvironment ${ }^{30}$. Substantial evidence indicates that macrophages, rather than being tumoricidal as suggested after their activation in vitro (Fidler, 1988), adopt a protumoral phenotype in vivo both in the primary and metastatic sites (Biswas et al., 2013). Indeed in lung cancer, macrophages are polarized to a protumoral phenotype at the time of tumor initiation (Redente et al., 2010). These activities include suppression of $\mathrm{T}$ cell responses (Coussens et al., 2013; Qian and Pollard, 2010). In addition, macrophages promote many important features of tumor progression including angiogenesis, tumor cell invasion, motility, and intravasation as well as at the metastatic site, stimulation of tumor cell extravasation and persistent growth (Qian and Pollard, 2010) $)^{30,31}$.

Tumor-Associated Macrophages in the Primary Tumor Promote Malignancy In the primary tumor, microenvironment macrophages under the influence of IL-4 produced by CD4+ T cells and tumors and WNT7b promote tumor cell invasion. This invasion is mediated via a paracrine loop involving tumor-synthesized CSF1 and macrophage-produced EGF that drives migration of tumor cells in lock-step with macrophages along collagen fibers that act as highways toward blood 
vessels $^{31}$. This process also requires TGFb that drives an epithelial-mesenchymal transition (EMT) in the tumor cells that promotes migration and matrix remodeling via Cathepsins and matrix adhesion of tumor cells via SPARC. This streaming of tumor cells results in their pileup on the vessels where macrophages promote their intravasation into the circulation through a structure named the "Tumor Microenvironment of Metastasis" (TMEN). In addition to effect on tumor cell migration and invasion, TIE2+ macrophages produce VEGF and WNT7b that stimulates angiogenesis in the tumor. Thus, there is an additive effect caused by macrophages of increased migration of tumor cells toward vessels and increased vascular targets that results in a large number of circulating tumor cells and thus increased malignancy ${ }^{32}$.

The role of tumor-associated macrophages in human skin cancer

Inflammation is recognized as being important for tumorigenesis and was recently proposed as the seventh hallmark of cancer ${ }^{33}$. The inflammatory infiltrate in tumors most commonly consists predominantly of macrophages, T cells, neutrophils, natural killer (NK) cells - but includes other hematopoietic cells ${ }^{34}$. Among these cells, macrophages are the most numerous cells in this tumor infiltrate. Tumor-associated macrophages (TAMs) are closely involved in multiple stages of carcinogenesis: they contribute to initiation, growth, invasion, and metastasis of tumors through production of a diverse array of cytokines, growth factors, proangiogenic factors, and matrix metalloproteinases ${ }^{35}$ the most common cancer in the United States is nonmelanoma skin cancer with an estimated 1.3 million cases each year. The incidence of NMSC is increasing worldwide. It is essential to understand macrophage phenotypic characteristics in tumours of diverse clinical behaviour to understand which factors correlate with tumor growth, invasion and metastasis. The two most common forms of NMSC are basal cell carcinoma (BCC) and squamous cell carcinoma (SCC). BCC and SCC are ideal tumors to consider in study of TAMs in humans as they represent two common epidermal neoplasms that behave differently clinically. BCCs only grow and invade locally and do not metastasize. In contrast, SCCs are more invasive and can metastasize in late stages. Sun exposure, specifically UV-B radiation, is considered to be one of the most important environmental factors involved in initiation of SCC. UV-B exposure directly leads to DNA and RNA damage through formation of pyrimidine dimers ${ }^{36}$. A limited number of studies have examined the role of macrophages in skin cancer. In BCC, it has been shown recently that the number of TAMs infiltrating the tumor directly correlates with depth of tumor invasion and microvessel density. In cutaneous SCC, TAMs are shown to be a major source of VEGF-C, a critical lymphangiogenic factor ${ }^{37}$. Therefore, study of in vivo TAMs in SCC and BCC represents a suitable strategy for further understanding of cutaneous carcinogenesis. TAMs classically exhibit two states of activation known as M1 and M2 states. Macrophages are polarized in response to Th1 cytokines, LPS and IFN-gamma, into an M1 state. On the other hand, in response to Th2 cytokines, IL-4 and IL-13, macrophages enter an M2 state. M1 macrophages are inflammatory and produce a variety of pro-inflammatory factors such as IL-1 $\beta$, TNF$\alpha$, and IL-6. In general, M1 macrophages are involved in clearance of microorganisms ${ }^{38}$. M2 macrophages on the other hand are involved in type 2 inflammation, tissue remodelling, angiogenesis, and are believed to promote tumor growth. M2 macrophages produce high levels of Interleukin-10 (IL-10) and arginase I and express numerous scavenger receptors. The classical M1-M2 paradigm has been recently revealed to represent two ends of a spectrum that can also include heterogeneous states of activation. It has been shown in multiple studies that macrophages can show mixed states of activation and there are multiple sub-populations of $\mathrm{TAMs}^{39}$.

Epithelial skin cancers, in particular basal and squamous cell carcinomas (BCC and SCC), are the most frequent types of cancer in humans, and they are diagnosed in 23 million people worldwide every year ${ }^{40}$. Due to the increased life expectancy, the enhanced sun exposure, and the treatment of organ transplant patients with immunosuppressive compounds, the incidence of epithelial skin cancer is continuously increasing (Griffin et al, 2016). Cutaneous SCCs often develop from precursor lesions, of which actinic keratosis (AK) is the most frequent one. Thus, it has been estimated that $65 \%$ of cutaneous SCCs arise from AK (Criscione et al, 2009) $^{41,42}$. AK develops at multiple sites, in particular in sun-exposed skin, and its treatment is important due to the risk of malignant transformation (Werner et al, 2013). Importantly, it is generally difficult to predict whether an AK lesion will progress to SCC, and it is therefore important to identify biomarkers that indicate a high risk for malignant progression. This requires a thorough understanding of the pathomechanisms underlying $\mathrm{AK}$ development and progression and the identification and functional characterization of the involved cell types and genes/proteins. Some of them may represent novel targets for therapeutic intervention. They include proteins that directly affect proliferation, survival and migration of keratinocytes and their malignant transformation, but also proteins that act on various components of the tumor stroma. Among the latter is the growth and differentiation factor activin, a member of the transforming growth factor $\beta$ (TGF- $\beta$ ) family. Activins are homo- or heterodimers composed of two $\beta$ chains, with activin $A(\beta A \beta A)$ being the most abundant and best-characterized variant (Chen et al, 2006) ${ }^{39}$. The activity of activins is inhibited through binding to the secreted glycoproteins follistatin or follistatin-related protein in the extracellular environment (Xia \& Schneyer, 2009). Activins signal via heterotetrameric receptor complexes consisting of type I and type II receptors, which are transmembrane serine/threonine kinases (Chen et al, 2006) ${ }^{43}$.

We previously showed that activin is strongly upregulated in murine and human skin wounds and in human BCCs and SCCs (Hubner et al, 1996; Antsiferova et al, 2011) ${ }^{44}$. This is functionally important, since overexpression of activin in 
keratinocytes of transgenic mice accelerated the wound healing process (Munz et al, 1999), but also promoted skin carcinogenesis and malignant progression of existing tumors in a murine skin cancer model where tumors are induced by a combination of the mutagen 7,12-dimethylbenzo(a)anthracene (DMBA) and the tumor promoter 12-O-tetradecanoylphorbol-13-acetate

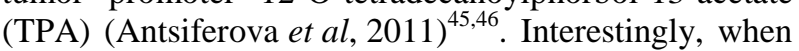
the activin-overexpressing mice were mated with transgenic mice expressing a dominant-negative activin receptor mutant in keratinocytes, tumor development was not inhibited, but rather mildly increased. These findings demonstrate that activin does not promote tumorigenesis via keratinocytes, but rather has a mild tumor-suppressive effect on these cells. By contrast, activin obviously potentiates the oncogenic action of DMBA/TPA by generation of a pro-tumorigenic microenvironment. The latter involves loss of tumor-suppressive $\gamma \delta \mathrm{T}$ cells in the epidermis and a concomitant increase in cutaneous $\alpha \beta \mathrm{T}$ cells and Langerhans cells (Antsiferova et al, 2011) ${ }^{4445}$. However, it is unknown whether these cells are indeed functionally involved in the tumor-promoting effect of activin, and whether activin has additional, and possibly even more important cellular targets. In addition, it remains to be determined whether activin promotes skin tumorigenesis in more physiologically relevant tumor models and whether it is required at an early or late stage of skin cancer development. Most importantly, the molecular targets of activin in the affected cells of the tumor microenvironment remain largely unknown ${ }^{46,47}$.

Macrophage infiltration and alternative activation during wound healing promote mek1-induced skin carcinogenesis

The notion that the local microenvironment is actively involved in tumor development has gained wide acceptance in recent years. Interactions between cancer cells and surrounding nonmalignant cells are crucial determinants of cancer development ${ }^{47}$. It has been suggested that the construction of a pre-cancerous niche is required for initiated cancer cells to survive and evolve into a tumor. This niche is typically shaped by resident or recruited stromal cells, bone marrow derived cells, and signals or secreted factors (cytokines, chemokines, and exosomes) from all these populations .We have previously described a mouse model of wound induced, inflammation-mediated skin tumorigenesis ${ }^{48}$. In this model nondividing cells in the suprabasal layers of the epidermis express constitutively active MEK1 (InvEE transgenics), resulting in epidermal hyperproliferation and a chronic inflammatory skin infiltrate. Between $40 \%$ and $60 \%$ of InvEE transgenics develop benign tumors (papillomas and keratoacanthomas) at the site of a full-thickness circular wound, several days after the wound has healed. In this model MEK1 expressing, nondividing cells stimulate keratinocytes in the basal layer to divide and form the proliferative compartment of the tumour. MEK1 expressing suprabasal cells recruit an inflammatory infiltrate via secretion of pro-inflammatory cytokines including IL1a, which in turn induces expression of CD26 on stromal fibroblasts ${ }^{49}$. The presence of an immune infiltrate in InvEE skin is essential for woundinduced tumor formation, as treatment with the broadspectrum anti-inflammatory drug dexamethasone protects against tumor formation. Depletion of circulating gd $\mathrm{T}$ cells, but not ab $\mathrm{T}$ cells or $\mathrm{B}$ cells, reduces the incidence of tumors and leads to a reduction in the number of skin macrophages. Depletion of skin macrophages by treatment of the wound site with clodronate liposomes also decreases tumor incidence ${ }^{50}$, 51 . The identification of macrophages as key contributors to InvEE tumor formation is consistent with studies in a wide variety of other tumor types. Macrophages are highly specialized to support established neoplastic lesions, by promoting immunosuppression, proliferation, angiogenesis, and metastasis ${ }^{52,53}$. Correlations between persistent, macrophage mediated chronic inflammation and cancer have been noted ${ }^{54,55}$. However, little is known about the role of macrophages in the earliest stages of tumor development. Initiating events, such as tissue or DNA damage caused by the release of cytotoxic factors through inflammatory cells, are difficult to identify as they may occur long before overt tumor development and in most experimental systems the precise site of the tumor cannot be predicted. Our model allows the observation of events that ultimately lead to tumor formation in a well-defined pre-cancerous niche, the wound ${ }^{56,57}$.

\section{Macrophage polarization in inflammatory diseases}

Macrophages were first identified by Elie Metchnikoff as phagocytic cells which helped to liminate pathogens in both invertebrates and vertebrates. In 1905, his research findings suggested that macrophages from infected animals had elevated ability of killing bacteria, thereby proposing the basis of the concept of macrophage activation ${ }^{59,60}$. After six decades of efforts, the mechanisms with regard to killing bacteria of macrophages were gradually elucidated, but there were still no definite answers about how macrophages became more efficient bacterial k illers. In 1973, North and his colleagues found that independent cellular factors could also promote resistance of infection without involvement of pathogens Almost at the same time, David indicated that lymphocytes were the major antigen-specific cells responsible for microbicidal activation of macrophages ${ }^{61,} 62$.Soon after that, interferon (IFN)- $\gamma$, produced by lymphocytes, was identified as the firstfactor for interaction between macrophages and lymphocytes ${ }^{59}$. It transforms resting macrophagesinto active ones which have stronger antigen presenting capacity and complement mediated phagocytosis,and secrete more pro-inflammatory cytokines as well as toxic mediators. As the first type of antimicrobialmacrophage activation was recognized, it became known as classically activated macrophages (CAM, also known as M1). In 1989, when the heterogeneity in the helper T-cell compartment was subsequently reported, Mosrnann and Coffman reviewed the different functions and lymphokines secretion between two types of cloned helper $\mathrm{T}$ cells (Th), and proposed the concept of Th1 and $\mathrm{Th} 2^{63,64}$.

colleagues recognized that interleukin (IL)-4, which was mainly produced by Th2 cells, could convert 
macrophages into a special activation state compared with IFN- $\gamma$ induced activation in which respiratory burst was inhibited and major histocompatibility complex class II antigens (MHC-II) expression was increased 65,66 . With the discovery of up-regulation of macrophage mannose receptor (MRC1) as a specific marker of IL4/IL-13-activated macrophages in 1992, which was coupled with the enhanced expression of MHC-II, the concept of alternatively activated macrophages (AAM, also known as M2) was first proposed . In the following years, when the plasticity of macrophages in response to different environment was gradually studied, Mosser and Edwards reviewed the full spectrum of macrophage activation and pointed out that M1 and M2 were two terminals of the spectrum ${ }^{67,68}$.

\section{Atherosclerosis and cardiovascular diseases}

Atherosclerosis is a common type of degenerative disease of the vessel wall characterized by the accumulation of apolipoprotein B-lipoproteins in the inner lining of large and medium sized arteries ${ }^{69}$. It underlies the leading cause of death in developed countries and is likely soon to attain this status worldwide. Monocytes and macrophages play essential roles in the development of atherosclerosis. As the apolipoprotein B-lipoproteins accumulated, the endothelial cells become dysfunction and secrete a sum of chemokines, which interact with receptors on the circulating monocytes and promote them into the vessel wall. Those monocytes then transform into macrophages and take up cholesterol to give rise to a structure called atherosclerotic plaque. As diseases develop, atherosclerotic plaque can grow larger, even become vulnerable and rupture, potentially resulting in a heart attack, stroke and even sudden cardiac death ${ }^{70}$. The fact that prevention of monocyte recruitment by blocking chemokines or their receptors could inhibit or slow down atherogenesis in mouse model of atherosclerosis, might provide strong support for the essential role of macrophages in the development of atherosclerosis ${ }^{65}$.In patients with unstable angina and myocardial infarction, the pro-inflammatory cytokines secreted by M1 phenotype macrophages were elevated, such as IL-6, with high levels predicating a poor outcome ${ }^{71}$. An in vitro study indicated that M1 phenotype macrophages could also induce smooth muscle cell proliferation and release of vaso active molecules including NO, endothelins as well as eicosanoids, and they were important consequences for lipoprotein oxidation and cytotoxicity. Early atherosclerotic plaques were infiltrated by M2 phenotype macrophages, however, along with the progression of the plaques, M1 phenotype macrophages gradually increased and occupied a major position, there by more likely leading to an acute a the thrombotic vascular accident ${ }^{72}$.

\section{Obesity and insulin resistance}

Obesity and its attendant metabolic disorder challenge the public health of modern society worldwide. Nearly 75\% adults in America are overweight, and more than one-third of them are obese. Furthermore, the persistent increase in obesity, especially in children, will halt or even decrease the life expectancy of America within the first half of this century ${ }^{73,74}$. As a disease with metabolic disturbance, obesity could lead to insulin resistance, glucose intolerance dyslipidemia as well as hypertension. In addition, recent studies had uncovered that obesity was involved in cancers, hepatic and renal failure, thrombotic disease, and many infectious diseases ${ }^{75}$. Those findings suggest that obesity should pay more attentions than ever before. Substantial evidences demonstrate that obesity is a chronic lowgrade inflammatory disease. An important initiator of the inflammatory reaction to obesity is adipose tissue, which is consisting of adipocytes, preadipocytes, endothelial cells and immune cells (e.g., macrophages and lymphocytes). In obesity, adipocytes can release pro-inflammatory mediators, such as $\mathrm{CC}$ chemokine ligand (CCL)-2, TNF- $\alpha$, free fatty acids (FFAs), instead of leptin and a diponectin, which promote insulin sensitivity in normal state ${ }^{76,77}$. Those pro-inflammatory mediators induce the recruitment and activation of adipose tissue macrophage (ATM). The activated ATM secrets pro-inflammatory cytokines and forms the inflammatory circuit which blocks the insulin action of a dipocytes and leads to insulin resistance ${ }^{78,79}$.

\section{Tumor}

It is widely accepted that smouldering inflammation is an essential cause of many cancers, and has been suggested as the 7th hallmark of cancer 80 . Similar to other chronic inflammation, cancer-related inflammation is also mediated by inflammatory mediators (chemokines, cytokines, and prostaglandins) and inflammatory cells, constituting a microenvironment for the initiation, growth and metastasis ofcancers ${ }^{81}$. Tumorassociated macrophages (TAM), the major component of those inflammatory cells infiltrated in cancer, play critical roles in the formation of cancer. In the stage of tumor initiation, TAM releases a large amount of inflammatory mediators to create an inflammatory environment and promote tumor growth. When tumors progress to malignancy, TAM contributes to the angiogenesis, invasion, metastasis of tumors, and decreases the anti-tumor immunity. As tumors become metastatic, TAM infiltrates the target tissues and makes beds for the arrival of tumor cells. In addition, some TAM even changes its phenotype to help extravasation, survival, and subsequent growth of tumor cells ${ }^{82}$. It has been demonstrated that there is a strong link between poor prognosis and increased TAM in thyroid, lung, and hepatocellular tumors. A recent experiment showed that an increased number of TAM was strongly associated with a shortened survival in classical Hodgkin's lymphoma patients. Therefore, the population of TAM could be a biomarker for the risk stratification in tumors 83 .

\section{Asthma}

Asthma is a chronic inflammatory disease which is wide-spread in both developing and developed countries. Disorder in the regulation of phenotypes of pulmonary macrophages contributes to the pathogenesis of asthma. It is widely accepted that M2 phenotype macrophages take a leading role in asthma, which they are beneficial for tissue repairing and restoration of 
homeostasis in the microenvironment of lung tissue. However, excessive M2 macrophages may increase cell recruitment and mucus secretion, and result in airway hyper-responsiveness ${ }^{84,85}$. Moreira et al transferred M2 phenotype macrophages into the lungs of fungusinduced asthmatic mice, then, both the inflammatory response and collagen deposition were enhanced, in turn accelerating the pathophysiological process of asthma ${ }^{86}$. With a boom in research of macrophage polarization, growing evidences support that M1 phenotype macrophages contribute to the development of asthma. In severe forms of asthma, especially in patients resistant to glucocorticoid therapy, macrophages are shown to become an M1 phenotype, which produces a great amount of pro-inflammatory mediators, including TNF- $\alpha$, IL-1 $\beta$, NO, exacerbates the lung injury and accelerate the airway remodeling For instance, NO produced by M1 phenotype leads to oxidative DNA damage and inflammation, enhances mucus production,

\section{REFERENCES:}

1. Metchnikoff E. Immunity in the Infectious Diseases. New York: Macmillan. 1905.

2. Zhou, D.; Huang, C.; Lin, Z.; Zhan, S.; Kong, L.; Fang, C.; Li, J. Macrophage polarization and function with emphasis on the evolving roles of coordinated regulation of cellular signalling pathways. Cell. Signal. 2014, 26, 192-197.

3. North RJ. Cellular mediators of anti-Listeria immunity as an enlarged population of short lived, replicating T cells. Kinetics of their production. The Journal of experimental medicine. 1973; 138: 342-55.

4. Neumann, N.F.; Stafford, J.L.; Belosevic, M. Biochemical and functional characterisation of macrophage stimulating factors secreted by mitogen-induced goldfish kidney leucocytes. Fish Shellfish Immunol. 2000, 10, 167-186.

5. Rieger, A.M.; Hall, B.E.; Barreda, D.R. Macrophage activation differentially modulates particle binding, phagocytosis and downstream antimicrobial mechanisms. Dev. Comp. Immunol. 2010, 34, 1144-1159.

6. Grayfer, L.; Hodgkinson, J.W.; Belosevic, M. Antimicrobial responses of teleost phagocytes and innate immune evasion strategies of intracellular bacteria. Dev. Comp. Immunol 2014, 43, 223-242.

7. Joerink, M.; Savelkoul, H.F.J.; Wiegertjes, G.F. Evolutionary conservation of alternative activation of macrophages: Structural and functional characterization of arginase 1 and 2 in carp (Cyprinus carpio L.). Mol. Immunol. 2006, 43, 11161128.

8. Takizawa, F.; Koppang, E.O.; Ohtani, M.; Nakanishi, T.; Hashimoto, K.; Fischer, U.; Dijkstra, J.M. Constitutive high expression of interleukin-4/13A and GATA-3 in gill and skin of salmonid fishes suggests that these tissues form Th2 skewed immune environments. Mol. Immunol. 2011, 48, 1360-1368.

9. Castro, R.; Zou, J.; Secombes, C.J.; Martin, S.A.M. Cortisol modulates the induction of inflammatory gene expression in a rainbow trout macrophage cell line. Fish Shellfish Immunol. 2011, 30, 215-223.

10. Grayfer, L.; Hodgkinson, J.W.; Hitchen, S.J.; Belosevic, M. Characterization and functional analysis of goldfish (Carassius auratus L.) interleukin-10. Mol. Immunol. 2011, 48, 563-571.

11. Joerink, M.; Forlenza, M.; Ribeiro, C.M.S.; de Vries, B.J.; Savelkoul, H.F.J.; Wiegertjes, G.F. Differential macrophage polarisation during parasitic infections in common carp (Cyprinus carpio L.). Fish Shellfish Immunol. 2006, 21, 561571.

12. Gautier EL, et al. Gene-expression profiles and transcriptional regulatory pathways that underlie the identity and diversity of mouse tissue macrophages. Nat Immunol. 2012; 13:11181128. [PubMed: 23023392]. and amplifies the lung injury in murine model of allergen-induced airway disease ${ }^{87}$.

\section{CONCLUSIONS}

Macrophages have numerous different functions that may be injurious or reparative. Key issues for the future to devise a more complete understanding of the range of macrophage activities. In this review, we include all the that activin overexpression is an early event in murine and human skin carcinogenesis and that increased levels of activin strongly promote formation of skin tumors induced by the human papillomavirus 8 (HPV8). We provide functional evidence for a crucial role of macrophages in the pro-tumorigenic effect of activin. Finally, we provide the first genome-wide RNA profiling data of macrophages isolated from pre-tumorigenic lesions, which identified novel activin target genes and revealed that activin induces a pro-tumorigenic macrophage phenotype.

13. Gordon S. Alternative activation of macrophages. Nat Rev Immunol. 2003; 3:23-35. [PubMed: 12511873]

14. Sica A, Mantovani A. Macrophage plasticity and polarization: in vivo veritas. The Journal of clinical investigation. 2012; 122:787-795. [PubMed: 22378047].

15. Geissmann F, et al. Development of monocytes, macrophages, and dendritic cells. Science. 2010; 327:656-661. [PubMed: 20133564].

16. Jenkins SJ, et al. Local Macrophage Proliferation, Rather than Recruitment from te Blood, Is a Sinature of Th2 Inflammation. Science. 2011.

17. Schulz C, et al. A lineage of myeloid cells independent of Myb and hematopoietic stem cells. Science. 2012; 336:86-90. [PubMed: 22442384].

18. Ohmori Y, Hamilton TA. IL-4-induced STAT6 suppresses IFN-gamma-stimulated STAT1-dependent transcription in mouse macrophages. The Journal of immunology. 1997; 159: 5474-82.

19. Satoh T, Takeuchi O, Vandenbon A, Yasuda K, Tanaka Y, Kamara Y, et al. The Jmjd3-Irf4 axis regulates M2 macrophage polarization and host responses against helminthic infection. Nature immunology. 2010; 11: 936-44. doi:10.1038/ni.1920.

20. Vishvakrama P, Sharma S. Liposomes: An Overview. Journal of Drug Delivery and Therapeutics, 2014; 0:47-55. doi:10.22270/jddt.v0i0.843

21. Oeckinghaus A, Hayden MS, Ghosh S. Crosstalk in NFkappaB signalling pathways. Nature immunology. 2011; 12: 695-708. doi:10.1038/ni.2065.

22. Dwivedi C, Sahu R, Tiwari S, Satapathy T, Roy A. Role of liposome in novel drug delivery system. Journal of Drug Delivery and Therapeutics, 2014; 4(2):116-129. doi:10.22270/jddt.v4i2.768

23. Janssen, Knol, Egestion, Macrophages and hematopoietic cell clusters from mouse bone marrow. J exp med. 1985; 162(3):993-1014.

24. Dantzer r, o'connor jc, freund gg, johnson rw, kelley kw. From inflammation to sickness and depression: when the immune system subjugates the brain. Nat rev neurosci. 2008; 9(1):46-56.

25. Lawson LJ, Perry VH, Dri P, Gordon S. Heterogeneity in the distribution and morphology of microglia in the normal adult mouse brain. Neuroscience. 1990; 39(1):151-70

26. Kitamura T, Qian Bz, Pollard Jw. Immune Cell Promotion of Metastasis. Nat Rev Immunol. 2015; 15(2):73-86.

27. Abraham, D., Zins, K., Sioud, M., Lucas, T., Scha“ fer, R., Stanley, E.R., and Aharinejad, S. (2010). Stromal cell-derived CSF-1 blockade prolongs xenograft survival of CSF-1negative neuroblastoma. Int. J. Cancer 126, 1339-1352. 
28. Adeegbe, D.O., and Nishikawa, H. Natural and induced T regulatory cells in cancer. Frontiers in immunology 2013; 4, 190.

29. Balkwill, F., Charles, K.A., and Mantovani, A. Smoldering and polarized inflammation in the initiation and promotion of malignant disease. Cancer Cell 2005; 7, 211-217.

30. Glasauer, S.M.K.; Neuhauss, S.C.F. Whole-genome duplication in teleost fishes and its evolutionary consequences. Mol. Genet. Genom. 2014, 289, 1045-1060.

31. Coussens, L.M., and Werb, Z. 2002. Inflammation and cancer. Nature 420:860- 867.

32. Pollard, J.W. 2004. Tumour-educated macrophages promote tumour progression and metastasis. Nat Rev Cancer 4:71-78.

33. Mantovani, A., Sozzani, S., Locati, M., Allavena, P., and Sica, A. 2002. Macrophage polarization: tumor-associated macrophages as a paradigm for polarized M2 mononuclear phagocytes. Trends Immunol 23:549-555.

34. Grivennikov, S.I., Greten, F.R., and Karin, M. 2010. Immunity, inflammation, and cancer. Cell 140:883-899.

35. Van Ravenswaay Claasen, H.H., Kluin, P.M., and Fleuren, G.J. 1992. Tumor infiltrating cells in human cancer. On the possible role of CD16+ macrophages in antitumor cytotoxicity. Lab Invest 67:166-174.

36. Karin, M., and Greten, F.R. 2005. NF-kappa B: linking inflammation and immunity to cancer development and progression. In Nat Rev Immunol. England. 749-759.

37. Qian, B.Z., and Pollard, J.W. 2010. Macrophage diversity enhances tumor progression and metastasis. In Cell. United States: 2010 Elsevier Inc. 39-51.

38. Wyckoff, J.B., Wang, Y., Lin, E.Y., Li, J.F., Goswami, S., Stanley, E.R., Segall, J.E., Pollard, J.W., and Condeelis, J. 2007. Direct visualization of macrophageassisted tumor cell intravasation in mammary tumors. In Cancer Res. United States. 2649-2656.

39. Alam, M., and Ratner, D. 2001. Cutaneous squamous-cell carcinoma. N Engl J Med 344:975-983. 10. Lomas, A., Leonardi-Bee, J., and Bath-Hextall, F. 2012.

40. A systematic review of worldwide incidence of nonmelanoma skin cancer. Br J Dermatol 166:1069- 1080

41. Neville, J.A., Welch, E., and Leffell, D.J. 2007. Management of nonmelanoma skin cancer in 2007. In Nat Clin Pract Oncol. England. 462-469

42. Criscione VD, Weinstock MA, Naylor MF, Luque C, Eide MJ , Bingham SF, Department of Veteran Affairs Topical Tretinoin Chemoprevention Trial G, Actinic keratoses: natural history and risk of malignant transformation in the Veterans Affairs Topical Tretinoin Chemoprevention Trial. Cancer 2009; 115: 2523-2530.

43. Chen YG, Wang Q, Lin SL, Chang CD, Chuang J, Ying SY, Activin signaling and its role in regulation of cell proliferation, apoptosis, and carcinogenesis. Exp Biol Med (Maywood) 2006; 231: 534-544.

44. Antsiferova M, Huber M, Meyer M, Czuchra A, Ramadan T, MacLeod AS, HavranWL, Dummer R, Hohl D, Werner S, Ac tivin enhances skin tumourigenesis and malignant progression by inducing a pro-tumourigenic immune cell response. Nat Commun 2011; 2: 576.

45. Antsiferova $\mathrm{M}$, Werner $\mathrm{S}$, the bright and the dark sides of activin in wound healing and cancer. J Cell Sci 2012; 125: 3929-3937

46. Arwert EN, Lal R, Quist S, Rosewell I, van Rooijen N, Watt FM, Tumor formation initiated by nondividing epidermal cells via an inflammatory infiltrate. Proc Malt Acad Sci USA 2010; 107: 19903-19908

47. Basile JR, Holmbeck K, Bugge TH, Gutkind JS, MT1-MMP controls tumor-induced angiogenesis through the release of semaphorin 4D. J Biol Chem 2007; 282: 6899-6905

48. Bolger AM, Lohse M, Usadel B, Trimmomatic: a flexible trimmer for Illumina sequence data. Bioinformatics 30: 2114

49. Musawi SL, Kelly EK, Qian H, La M, Lu L, Lovrecz G, Ziem ann M, Lazarus R et al, Development of novel activintargeted therapeutics. Mol Ther 2015; 23:434-444

50. Daniel D, Meyer Morse N, Bergsland EK, Dehne K, Coussens LM, Hanahan D, Immune enhancement of skin carcinogenesis by $\mathrm{CD}^{+}{ }^{+} \mathrm{T}$ cells. J Exp Med 2003; 197: 10171028.

51. .Egeblad M, Nakasone ES, Werb Z. Tumors as organs: complex tissues that interface with the entire organism. Dev Cell 2010; 18:884-901.

52. Elinav E, Nowarski R, Thaiss CA, Hu B, Jin C, Flavell RA. Inflammationinduced cancer: crosstalk between tumours, immune cells and microorganisms. Nat Rev Cancer 2013; 13:759-71.

53. Pietras K, Ostman A. Hallmarks of cancer: interactions with the tumor stroma. Exp Cell Res 2010; 316:1324-31.

54. Barcellos-Hoff MH, Lyden D, Wang TC. The evolution of the cancer niche during multistage carcinogenesis. Nat Rev Cancer 2013; 13:511-8.

55. Arwert EN, Hoste E, Watt FM. Epithelial stem cells, wound healing and cancer. Nat Rev Cancer 2012; 12:170-80.

56. Arwert EN, Lal R, Quist S, Rosewell I, van Rooijen N, Watt FM. Tumor formation initiated by nondividing epidermal cells via an inflammatory infiltrate. Proc Natl Acad Sci U S A 2010; 107:19903-8.

57. Hobbs RM, Silva-Vargas V, Groves R, Watt FM. Expression of activated MEK1 in differentiating epidermal cells is sufficient to generate hyperproliferative and inflammatory skin lesions. J Invest Dermatol 2004; 123:503-15.

58. Pollard JW. Tumour-educated macrophages promote tumour progression and metastasis. Nat Rev Cancer 2004; 4:71-8.

59. Balkwill F, Charles KA, Mantovani A. Smoldering and polarized inflammation in the initiation and promotion of malignant disease. Cancer Cell 2005; 7:211-7.

60. Grivennikov SI, Greten FR, Karin M. Immunity, inflammation, and cancer. Cell 2010; 140:883-99.

61. North RJ. Cellular mediators of anti-Listeria immunity as an enlarged population of short lived, replicating T cells. Kinetics of their production. The Journal of experimental medicine. 1973; 138: 342-55.

62. David JR. Lymphocyte mediators and cellular hypersensitivity. The New England journal of medicine. 1973; 288: 143-9. Doi: 10.1056/NEJM197301182880311.

63. Nathan CF, Murray HW, Wiebe ME, Rubin BY. Identification of interferon-gamma as the lymphokine that activates human macrophage oxidative metabolism and antimicrobial activity. The Journal of experimental medicine. 1983; 158: 670-89.

64. Mosmann TR, Coffman RL. TH1 and TH2 cells: different patterns of lymphokine secretion lead to different functional properties. Annual review of immunology. 1989; 7: 145-73. doi:10.1146/annurev.iy.07.040189.001045.

65. Abramson SL, Gallin JI. IL-4 inhibits superoxide production by human mononuclear phagocytes. The Journal of immunology. 1990; 144: 625-30.

66. Stein M, Keshav S, Harris N, Gordon S. Interleukin 4 potently enhances murine macrophage mannose receptor activity: a marker of alternative immunologic macrophage activation. The Journal of experimental medicine.1992; 176: 287-92.

67. Moore KJ, Tabas I. Macrophages in the pathogenesis of atherosclerosis. Cell. 2011; 145: 341-55. doi:10.1016/j.cell.2011.04.005.

68. Lloyd-Jones D, Adams RJ, Brown TM, Carnethon M, Dai S, and De Simone G, et al. Executive summary: heart disease and stroke statistics--2010 update: a report from the American Heart Association. Circulation. 2010; 121: 948-54.

69. Swirski FK, Nahrendorf M. Leukocyte behavior in atherosclerosis, myocardial infarction, and heart failure. Science. 2013; 339: 161-6. doi:10.1126/science.1230719.

70. Fuster JJ, Fernandez P, Gonzalez-Navarro H, Silvestre C, Nabah YN, Andres V. Control of cell proliferation in atherosclerosis: insights from animal models and human studies. Cardiovascular research. 2010; 86: 254-64. doi:10.1093/cvr/cvp363.

71. Mestas J, Ley K. Monocyte-endothelial cell interactions in the development of atherosclerosis. Trends in cardiovascular medicine. 2008; 18: 228-32.doi:10.1016/j.tcm.2008.11.004.

72. Kirbis S, Breskvar UD, Sabovic M, Zupan I, Sinkovic A. Inflammation markers in patients with coronary artery disease-comparison of intracoronary and systemic levels. Wiener 
klinische Wochenschrift. 2010; 122 Suppl 2: 31-4. Doi: 10.1007/s00508-010-1343-z.

73. Khallou-Laschet J, Varthaman A, Fornasa G, Compain C, Gaston AT, Clement M, et al. Macrophage plasticity in experimental atherosclerosis. PloS one. 2010; 5: e8852. doi:10.1371/journal.pone.0008852.

74. Tsimikas S, Miller YI. Oxidative modification of lipoproteins: mechanisms, role in inflammation and potential clinical applications in cardiovascular disease. Current pharmaceutical design. 2011; 17: 27-37

75. Handberg A, Skjelland M, Michelsen AE, Sagen EL, KrohgSorensen K, Russell D, et al. Soluble CD36 in plasma is increased in patients with symptomatic atherosclerotic carotid plaques and is related to plaque instability. Stroke, a journal of cerebral circulation. 2008; 39: 3092-5. doi:10.1161/STROKEAHA.108.517128.

76. Huo Y, Zhao L, Hyman MC, Shashkin P, Harry BL, Burcin T, et al. Critical role of macrophage 12/15-lipoxygenase for atherosclerosis in apolipoprotein E-deficient mice. Circulation. 2004; 110: 2024-31. doi:10.1161/01.CIR.0000143628.37680.F6.

77. Thorp E, Tabas I. Mechanisms and consequences of efferocytosis in advanced atherosclerosis. Journal of leukocyte biology. 2009; 86: 1089-95. doi:10.1189/jlb.0209115.

78. Olshansky SJ, Passaro DJ, Hershow RC, Layden J, Carnes BA, Brody J, et al. A potential decline in life expectancy in the United States in the 21st century. The New England journal of medicine. 2005; 352: 1138-45. Doi: 10.1056/NEJMsr043743.

79. Nguyen KD, Qiu Y, Cui X, Goh YP, Mwangi J, David T, et al. alternatively activated macrophages produce catecholamines to sustain adaptive thermogenesis. Nature. 2011; 480: 104-8. Doi: 10.1038/nature10653.

80. Mantovani A. Cancer: Inflaming metastasis. Nature. 2009; 457: 36-7. Doi: 10.1038/457036b.
81. Wei Q, Fang W, Ye L, Shen L, Zhang X, Fei X, et al. Density of tumor associated macrophage correlates with lymph node metastasis in papillary thyroid carcinoma. Thyroid: official journal of the American Thyroid Association. 2012. doi:10.1089/thy.2011-0452.

82. Shirabe $\mathrm{K}$, Mano $\mathrm{Y}$, Muto J, Matono R, Motomura $\mathrm{T}$, Toshima T, et al. Role of tumor-associated macrophages in the progression of hepatocellular carcinoma. Surgery today. 2012; 42: 1-7. Doi: 10.1007/s00595-011-0058-8.

83. Gocheva V, Wang HW, Gadea BB, Shree T, Hunter KE, Garfall AL, et al. IL-4 induces cathepsin protease activity in tumor-associated macrophages to promote cancer growth and invasion. Genes \& development. 2010; 24: 241-55. doi:10.1101/gad.1874010

84. Kitamura T, Kometani K, Hashida H, Matsunaga A, Miyoshi $\mathrm{H}$, Hosogi $\mathrm{H}$, et al. SMAD4-deficient intestinal tumors recruit CCR1+ myeloid cells that promote invasion. Nature genetics. 2007; 39: 467-75. Doi: 10.1038/ng1997.

85. Moreira AP, Hogaboam CM. Macrophages in allergic asthma: fine-tuning their pro- and anti-inflammatory actions for disease resolution. Journal of interferon \& cytokine research: the official journal of the International Society for Interferon and Cytokine Research. 2011; 31: 485-91. doi:10.1089/jir.2011.0027.

86. Kim YK, Oh SY, Jeon SG, Park HW, Lee SY, Chun EY, et al. Airway exposure levels of lipopolysaccharide determine type 1 versus type 2 experimental asthma. The Journal of immunology. 2007; 178: 5375-82.

87. Naura AS, Zerfaoui M, Kim H, Abd Elmageed ZY, Rodriguez $\mathrm{PC}$, Hans $\mathrm{CP}$, et al. Requirement for inducible nitric oxide synthase in chronic allergen exposure-induced pulmonary fibrosis but not inflammation. The Journal of immunology. 2010; 185: 3076-85. doi:10.4049/jimmunol.0904214. 